\title{
Assignment of parentage by microsatellite analysis in the endangered Brachymystax lenok tsinlingensis (Salmonidae)
}

\author{
Jian Shao ${ }^{1,2,3}$, Wei Luo ${ }^{4}$, Qiwei Wei ${ }^{1,2, *}$, Feng Wang ${ }^{5}$, Wei Guo ${ }^{2}$, Huan Ye ${ }^{2}$, \\ Zhipeng $\mathrm{Chu}^{2}$, Jinming $\mathrm{Wu}^{2}$, Shuhuan Zhang ${ }^{2}$ \\ ${ }^{1}$ College of Fisheries, Huazhong Agricultural University, Wuhan 430070, PR China \\ ${ }^{2}$ Yangtze River Fisheries Research Institute, Chinese Academy of Fishery Sciences, Wuhan 430223, PR China \\ ${ }^{3}$ College of Animal Science, Guizhou University, GuiYang 550025, PR China \\ ${ }^{4}$ South China Sea Institute of Oceanology, Chinese Academy of Sciences, Guangzhou 510301, PR China \\ ${ }^{5}$ Yellow River Fisheries Research Institute, Chinese Academy of Fishery Sciences, Xi'an 710086, PR China
}

\begin{abstract}
We evaluated the use of microsatellite markers to identify parentage in Brachymystax lenok tsinlingensis Li, 1966, an endangered salmonid. Computer simulations showed that when no parent information was available, or when information was only available from 1 parent, then exclusion probabilities ranged from 23.6 to $45.1 \%$ and from 25.9 to $52.4 \%$, respectively; combined exclusion probabilities for the 8 loci investigated were 97.7 and $98.4 \%$, respectively. However, a breeding experiment with known parental and filial information resulted in $91.28 \%$ of progeny being exclusively assigned to their correct parent pair. Our data demonstrate that microsatellitebased parentage assignment is a reliable means with which to obtain information pertaining to genealogical relationships and could therefore benefit genetic conservation of this species.
\end{abstract}

KEY WORDS: Brachymystax lenok tsinlingensis - Microsatellites · Parentage assignment . Inbreeding depression

\section{INTRODUCTION}

The genus Brachymystax includes 4 species: $B$. lenok, B. savinovi, B. tumensis, and B. tsinlingensis (B. lenok tsinlingensis $\mathrm{Li}, 1966)$, which are landlocked coldwater salmonid fishes. They are thought to originate from Siberia and were introduced to the Yellow Sea and the Bohai Sea as a result of glacial advances during quaternary glaciation (Li 1984). Migration to the sea was prevented by deglaciation, which led to sealed off distributaries and isolated local populations. Consequently, fish from this genus are not distributed contiguously in mountain streams, leading to long-term isolation in northern China (Sun \& Fang 1984).

Unfortunately, populations of $B$. lenok tsinlingensis have been rapidly declining as a result of over-

${ }^{*}$ Corresponding author: weiqw@yfi.ac.cn exploitation, environmental pollution, and habitat fragmentation (Zhao \& Zhang 2009, Froese \& Pauly 2015). The species is listed as 'vulnerable' in the China Red Data Book of Endangered Animals, and is classified as a second-class state-protected wild animal in China (Yue \& Chen 1998). Since 2008, B. lenok tsinlingensis has received increasing levels of attention for conservation via artificial propagation programs for the sole purpose of restocking the natural population to a sustainable level.

In order to minimize the deleterious effects of inbreeding, however, it is vital to understand the genetic background of the fish before considering artificial propagation. Currently, genetic evaluation of relatedness for broodstock management in captivity has been studied for some salmonid species, e.g. Oncorhynchus nerka (Kozfkay et al. 2008, Kali-

(C) The authors 2017. Open Access under Creative Commons by Attribution Licence. Use, distribution and reproduction are unrestricted. Authors and original publication must be credited. 
nowski et al. 2012) and Hucho taimen (Zhang et al. 2010). However, genetic studies of B. lenok tsinlingensis are limited to a number of investigations relating to the mitochondrial genome and general phylogeny (Liu et al. 2015, Yu \& Kwak 2015). In order to maintain genetic diversity, optimal survival, and growth traits, the most appropriate practice is to isolate and raise the progeny of different family groups in separate tanks until they are large enough to be tagged physically. However, this approach takes up too much space and is labor intensive. Moreover, it introduces environmental effects common to full-sibling groups that can confound genetic effects when estimating breeding values and other phenotypic and genetic parameters (Herbinger et al. 1999). Parentage inference using highly polymorphic codominant genetic markers, such as microsatellites (also called simple sequence repeats, or SSRs), is becoming a widely used tool to assess genetic relationship in selective breeding and population conservation for aquaculture as a way to identify and maintain pedigrees (Munkres et al. 2007, Harrison et al. 2014, Luo et al. 2014a,b). The use of a microsatellitebased parentage assignment system would allow progeny from different families to be communally stocked and retrospectively assigned to family of origin.

The present study was conducted to assess the potential for microsatellite markers previously developed for B. lenok tsinlingensis (Wang et al. 2015) to assign correct parentage. First, computer simulations were used to predict how many loci were required to provide a satisfactory rate of assignment success. We then used known parentprogeny relationships to test the power of selected loci to correctly assign parentage under hatchery conditions.

\section{MATERIALS AND METHODS}

\section{Mating strategy and rearing}

All broodstock were reared at the Shitouhe River Reserve (Shanxi Province, China). A small piece of dorsal fin was collected from all Brachymystax lenok tsinlingensis breeders. Broodstock was com- posed of 10 females (F) and 30 males (M), and artificial propagation was carried out with a ratio of $1 \mathrm{~F}: 3$ M. Fertilized eggs from each full-sib family were hatched in individual polycarbonate tanks $(0.5 \times$ $0.5 \times 0.3 \mathrm{~m}, 75 \mathrm{l}$ capacity) containing water from mountain streams that had been pre-treated by precipitation and filtration in a sedimentation pond. Temperature was maintained at $11 \pm 1.5^{\circ} \mathrm{C}$ (mean \pm $\mathrm{SD}$ ). After hatching, fingerlings were separately sampled from each family, and the sample size depended on the availability of offspring in each family. In total, 172 individuals were collected from all families.

\section{DNA extraction and microsatellite analysis}

Genomic DNA was extracted from fins (parents) or whole bodies (offspring) by using an established saltextraction method with slight modifications (Aljanabi \& Martinez 1997). A total of 172 progeny and 40 broodstock were genotyped with 8 polymorphic microsatellite loci (Wang et al. 2015). After a preliminary verification by PCR-PAGE, these loci were found to be highly polymorphic and were steadily amplified with clear bands from DNA obtained from B. lenok tsinlingensis. Details of the selected microsatellite loci are listed in Table 1. Each forward primer was labeled with carboxyfluorescein (FAM), carboxy-Xrhodamine (ROX), or hexachloro fluorescein (HEX). All PCR amplifications were carried out in a $10 \mu \mathrm{l}$ volume containing $1 \mu \mathrm{l}$ of $10 \times$ buffer (with $\mathrm{Mg}^{2+}$ ) for Taq DNA polymerase, $100 \mu \mathrm{M}$ dNTP, $0.5 \mu \mathrm{l}$ primer pairs,

Table 1. Microsatellite makers and primers used for the analysis of DNA in. Ta: amplification temperature used for PCR; FAM: carboxyfluorescein; HEX: hexachlorofluorescein; ROX: carboxy-X-rhodamine

\begin{tabular}{|c|c|c|c|c|c|}
\hline Locus & Sequence $\left(5^{\prime}-3^{\prime}\right)$ & $\operatorname{Ta}\left({ }^{\circ} \mathrm{C}\right)$ & Repeats & Size (bp) & Label \\
\hline BLT23 & $\begin{array}{l}\text { F: AATGCTTATTCACGCGAGGT } \\
\text { R: ACACACAGCTTGGGACACAG }\end{array}$ & 59 & $(\mathrm{AGTT})_{13}$ & $237-253$ & HEX \\
\hline BLT31 & $\begin{array}{l}\text { F: TGGATGGGTGTTACAAGCAA } \\
\text { R: CAGATCTTGAGACAAAGAGCCA }\end{array}$ & $\mathrm{A}^{56}$ & $(\mathrm{GTTA})_{10}$ & $161-177$ & FAM \\
\hline BLT21 & $\begin{array}{l}\text { F: CATTAATCCATCCAACCATGC } \\
\text { R: ACATCCCTGCCTTCGAAAC }\end{array}$ & 60 & $(\mathrm{TCAT})_{10}$ & $129-149$ & ROX \\
\hline BLT5 & $\begin{array}{l}\text { F: TTGAAGTTGCCTTCTGGTCC } \\
\text { R: GGCCACACATGCAAAACAT }\end{array}$ & 60 & $(\mathrm{TAA})_{11}$ & $164-176$ & FAM \\
\hline BLT10 & $\begin{array}{l}\text { F: GACAACAGCTACAGGGCACA } \\
\text { R: GACCTGGCTCTGGGTGATAG }\end{array}$ & 60 & $(\mathrm{ATC})_{12}$ & $239-521$ & FAM \\
\hline Hbl668 & $\begin{array}{l}\text { F: AATGAAACCAGCTCATTGCC } \\
\text { R: CAAGTCCTTCCAAATGGTCC }\end{array}$ & 58 & $(\mathrm{TG})_{14}$ & $171-189$ & ROX \\
\hline BLT101 & $\begin{array}{l}\text { F: TGTAATGTCACACACGCACG } \\
\text { R: CCAGACCAGAGGGACTTCAA }\end{array}$ & 60 & $(\mathrm{CA})_{13}$ & $171-189$ & HEX \\
\hline Hbl116 & $\begin{array}{l}\text { F: TGAACAGACACTCACACAGGC } \\
\text { R: GTGTTTCAGCTGCTGCGTT }\end{array}$ & 59 & $(\mathrm{TG})_{11}$ & $160-170$ & HEX \\
\hline
\end{tabular}


1 U Taq DNA polymerase, and 50 ng genomic DNA. The PCR program involved $5 \mathrm{~min}$ at $95^{\circ} \mathrm{C}$, followed by 30 cycles of $30 \mathrm{~s}$ at $94^{\circ} \mathrm{C}, 30 \mathrm{~s}$ at optimized annealing temperature (Table 1), $30 \mathrm{~s}$ at $72^{\circ} \mathrm{C}$, and a final extension period at $72^{\circ} \mathrm{C}$ for $8 \mathrm{~min}$.

\section{Computer simulation analyses}

A computer simulation was carried out to examine the feasibility of parentage analysis and to estimate the number of loci required. In the simulation, we assumed that markers were in linkage equilibrium, and there were no mutations or transmission errors between parents and progeny. Computer simulations were conducted using the CERVUS 3.0 program (Kalinowski et al. 2007). The term Excl 1 probability represented the power of each locus, and the combined probability over loci to exclude a false candidate parent from a family when the genotypes of the offspring were unknown, while the Excl 2 probability was used when the genotype of 1 parent was known. For these simulations, it was assumed that the proposed breeding program consisted of mating 10 dams to any 1 of 50 candidate sires.

\section{Parentage identification with known parental and filial information}

In order to evaluate the reliability of the 8 microsatellite markers in a real breeding program, an experiment was conducted in which both parental and filial information was known. The most likely candidate parent had the highest log-of-odds score. The parameters for this simulation were 10000 replication cycles and a pool of 40 candidate parents that were genotyped. In order to determine the number of mismatches between known mothers and their offspring, an initial computer simulation was performed with the typing error rate set at $1 \%$.

\section{RESULTS}

\section{Computed simulation}

CERVUS computer simulations revealed that exclusion probabilities per locus ranged from 0.24 to 0.48 when no parental information was known (Excl 1), and from 0.26 to 0.52 when information for 1 parent

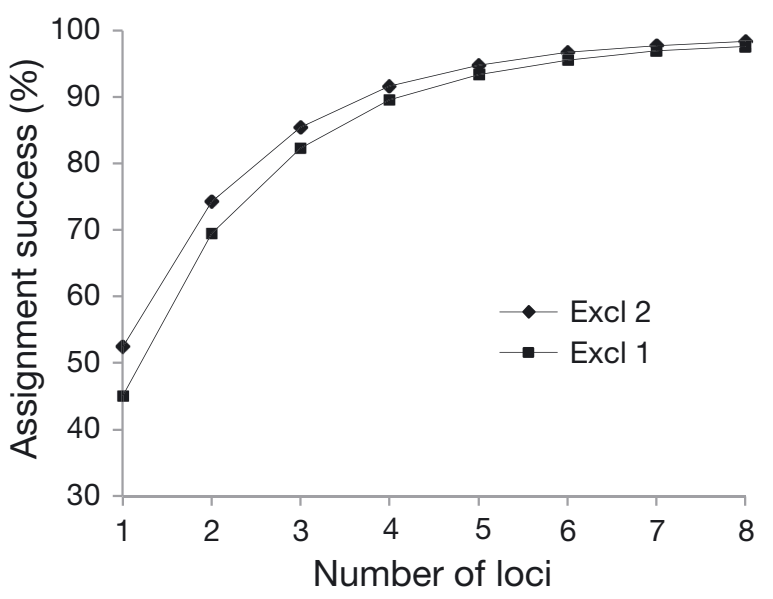

Fig. 1. Combined probabilities of exclusion derived from simulation studies and calculated over 8 selected polymorphic loci in Brachymystax lenok tsinlingensis, analyzed when no parental information was known (Excl 1) and when information for 1 parent was known (Excl 2)

was known (Excl 2). Combined probabilities of exclusion in the hypothetical population calculated for 8 polymorphic loci are shown in Fig. 1. Computer simulations demonstrated that at least 6 loci were required to assign $95 \%$ of progeny to both parents and 8 loci to assign $98 \%$ of progeny to both parents with acceptable confidence (greater than $95 \%$ of trials).

\section{Parentage identification with known parental and filial information}

The number of alleles detected from these 8 microsatellite loci ranged from 4 to 7 (Table 2). Mean values of expected heterozygosity and polymorphic information content were 0.69 and 0.61 , respectively.

Table 2. Probabilities of exclusion based either on the genotype of no known parentage (Excl 1) or when 1 parent was known (Excl 2) for 172 offspring of Brachymystax lenok tsinlingensis at 8 microsatellite loci. $k$ : number of alleles; $H_{\mathrm{e}}$ : expected heterozygosity; $H_{\mathrm{o}}$ : observed heterozygosity; PIC: polymorphic information content; HW: Hardy-Weinberg equilibrium

\begin{tabular}{|lcccccccc|}
\hline Locus & $k$ & $H_{\mathrm{e}}$ & $H_{\mathrm{o}}$ & PIC & Excl 1 & Excl 2 & HW & $\begin{array}{c}\text { Null } \\
\text { frequency }\end{array}$ \\
\hline BLT23 & 5 & 0.710 & 0.689 & 0.702 & 0.422 & 0.437 & 0.058 & +0.000 \\
BLT31 & 7 & 0.783 & 0.774 & 0.725 & 0.451 & 0.524 & 0.115 & +0.017 \\
BLT21 & 5 & 0.767 & 0.715 & 0.712 & 0.444 & 0.459 & 0.256 & -0.006 \\
BLT5 & 6 & 0.772 & 0.784 & 0.720 & 0.412 & 0.427 & 0.564 & +0.000 \\
BLT10 & 4 & 0.562 & 0.523 & 0.515 & 0.325 & 0.365 & 0.652 & +0.000 \\
Hbl101 & 5 & 0.687 & 0.664 & 0.642 & 0.366 & 0.378 & 0.254 & -0.009 \\
Hbl668 & 4 & 0.539 & 0.492 & 0.458 & 0.312 & 0.325 & 0.024 & +0.054 \\
Hbl116 & 6 & 0.679 & 0.645 & 0.613 & 0.236 & 0.259 & 0.231 & +0.000 \\
Average & 5.25 & 0.687 & 0.661 & 0.636 & 0.371 & 0.397 & & \\
\hline
\end{tabular}


Comparing mating design records and parentage assignment data determined by the 8 microsatellite markers, $96.5 \%$ and $93.6 \%$ of individuals were exclusively assigned to their true dam and sire, respectively. All but 15 of 172 offspring were exclusively assigned to their parent pair (assignment success rate was $91.3 \%$ ).

\section{DISCUSSION}

Many factors, including the number of microsatellite loci, their levels of polymorphism, and the number of potential pairings, affect the precision of assignment of an individual to a parental pair (Norris et al. 2000, Matson et al. 2008). In this study, the mean number of alleles was smaller than that reported for other fishes (Munkres et al. 2007, Li et al. 2010, López et al. 2012, Luo et al. 2014a). As a consequence, in our simulations, at least 6 microsatellite markers were needed to achieve $95 \%$ assignment success when there was information available regarding the parents. One possible explanation for this could be that a gradual reduction of genetic diversity has occurred in this species owing to inbreeding caused by several generations of domestication and artificial breeding (Estoup et al. 1998, Wang et al. 2015).

While previous computer simulations have provided important knowledge with regard to key parameters affecting parentage identification (Bernatchez \& Duchesne 2000, Norris et al. 2000), detailed experimental evaluation is still needed for individual species. In our study, 212 individuals (172 offspring and 40 parents) were genotyped for 8 selected microsatellite loci. These data demonstrated that this approach is a reliable method for parentage assignment and determining genealogical relationships in Brachymystax lenok tsinlingensis.

The real assignment success of the selected microsatellite markers to correctly assign parentage was lower than that obtained by computer simulations. Literature suggests that the presence of null alleles may represent a key source of errors in parentage assignment (Marshall et al. 1998, Li et al. 2010, Luo et al. 2014a). Four of the 8 selected loci presented null alleles, and the null allele frequency was $5.43 \%$ for locus Hbl668 (Table 2). Marshall et al. (1998) considered that pedigree determination was likely to be compromised if null allele frequencies exceeded $5 \%$. Thus, it was considered that this may have exerted at least some influence upon the accuracy of the assignments. Another reason for the lower assignment success observed in the real scenarios rather than the computer simulations may have been due to the existence of genotyping errors relating to allele mismatches at 1 or 2 loci (BLT31, Hbl668, or Hbl101). This accounted for an average of $8.33 \%$ at the 3 loci, a value higher than that reported for most other species of fish (Hoffman \& Amos 2005, Castro et al. 2007, Luo et al. 2014a). Thus, when choosing markers for parentage assignment, besides polymorphism and null allele frequency, the quality of the allelic pattern should also be taken into consideration since loci with a high number of stutter bands (or shadow bands) are often associated with high typing error rates (Estoup et al. 1998, López et al. 2012).

In conclusion, the microsatellite-based parentage assignment approach established here can replace physical tagging in order to obtain information regarding genealogical relationships among individuals over multiple generations, and thus to avoid inbreeding in stocks of $B$. lenok tsinlingensis. Consequently, this method represents a powerful tool for managing breeding programs and conserving the genetic resources of this endangered fish in the future.

Acknowledgements. This study was funded by the Special Fund for Agro-Scientific Research in the Public Interest from the Ministry of Agriculture of China (201203086), and Fundamental Research Funding of the Freshwater Fisheries Research Center, Chinese Academy of Fishery Sciences (2013JBFM19). We gratefully acknowledge Dengqiang Wang, Sien Wen, Bin Lu, Ping Li, and Xiaozhong Liu for their help during the experiment.

\section{LITERATURE CITED}

Aljanabi SM, Martinez I (1997) Universal and rapid saltextraction of high quality genomic DNA for PCR-based techniques. Nucleic Acids Res 25:4692-4693

* Bernatchez L, Duchesne P (2000) Individual-based genotype analysis in studies of parentage and population assignment: how many loci, how many alleles? Can J Fish Aquat Sci 57:1-12

* Castro J, Pino A, Hermida M, Bouza C and others (2007) A microsatellite marker tool for parentage assessment in gilthead seabream (Sparus aurata). Aquaculture 272(Suppl 1):S210-S216

Estoup A, Gharbi K, SanCristobal M, Chevalet C, Haffray P, Guyomard R (1998) Parentage assignment using microsatellites in turbot (Scophthalmus maximus) and rainbow trout (Oncorhynchus mykiss) hatchery populations. Can J Fish Aquat Sci 55:715-725

Froese R, Pauly D (2015) FishBase. www.fishbase.org (accessed 10 December 2015)

* Harrison HB, Feldheim KA, Jones GP, Mansour H, Perumal S, Williamson DH, Berumen ML (2014) Validation of microsatellite multiplexes for parentage analysis in a coral reef fish (Lutjanus carponotatus, Lutjanidae). Conserv Genet Resour 6:803-806 
Herbinger CM, O'Reilly PT, Doyle RW, Wright JM, O'Flynn F (1999) Early growth performance of Atlantic salmon full-sib families reared in single family tanks versus in mixed family tanks. Aquaculture 173:105-116

Hoffman JI, Amos W (2005) Microsatellite genotyping errors: detection approaches, common sources and consequences for paternal exclusion. Mol Ecol 14:599-612

Kalinowski ST, Taper ML, Marshall TC (2007) Revising how the computer program CERVUS accommodates genotyping error increases success in paternity assignment. Mol Ecol 16:1099-1106

Kalinowski ST, Van Doornik DM, Kozfkay CC, Waples RS (2012) Genetic diversity in the Snake River sockeye salmon captive broodstock program as estimated from broodstock records. Conserv Genet 13:1183-1193

Kozfkay CC, Campbell MR, Heindel JA, Baker DJ, Kline P, Powell MS, Flagg T (2008) A genetic evaluation of relatedness for broodstock management of captive, endangered Snake River sockeye salmon, Oncorhynchus nerka. Conserv Genet 9:1421-1430

Li R, Qi L, Cornette F, Dégremont L, Lapègue S (2010) Development of four EST-SSR multiplex PCRs in the Pacific oyster (Crassostrea gigas) and their validation in parentage assignment. Aquaculture 310:234-239

Li SZ (1984) Study on the distribution of the salmonid fishes in China. Dongwuxue Zazhi 3:34-37 (in Chinese)

* Liu H, Li Y, Liu X, Xiong D, Wang L, Zou G, Wei Q (2015) Phylogeographic structure of Brachymystax lenok tsinlingensis (Salmonidae) populations in the Qinling Mountains, Shaanxi, based on mtDNA control region. Mitochondrial DNA 26:532-537

Kópez A, Pardo BG, Planas M, Quintas P, Martínez P, Bouza C (2012) A microsatellite panel for mating system analysis and broodstock management of captive long-snouted seahorse Hippocampus guttulatus. Aquaculture 356-357: 153-157

Luo W, Zeng C, Deng W, Robinson N, Wang W, Gao Z (2014a) Genetic parameter estimates for growth-related traits of blunt snout bream (Megalobrama amblycephala) using microsatellite-based pedigree. Aquacult Res 45:1881-1888

Luo W, Zeng C, Yi S, Robinson N, Wang W, Gao Z (2014b)

Editorial responsibility: Thomas Turner,

Albuquerque, New Mexico, USA
Heterosis and combining ability evaluation for growth traits of blunt snout bream (Megalobrama amblycephala) when crossbreeding three strains. Chin Sci Bull 59: $857-864$

Marshall TC, Slate J, Kruuk LEB, Pemberton JM (1998) Statistical confidence for likelihood-based paternity inference in natural populations. Mol Ecol 7:639-655

Matson SE, Camara MD, Eichert W, Banks MA (2008) p-loci: a computer program for choosing the most efficient set of loci for parentage assignment. Mol Ecol Resour 8: 765-768

* Munkres KP, Bay LK, Jerry DR, McCormick MI, Herwerden LV (2007) Development and characterization of microsatellite markers for parentage analyses of the coral reef damselfish (Pomacentrus amboinensis: Pomacentridae). Conserv Genet 8:987-990

Norris AT, Bradley DG, Cunningham EP (2000) Parentage and relatedness determination in farmed Atlantic salmon (Salmo salar) using microsatellite markers. Aquaculture 182:73-83

Sun SL, Fang SM (1984) Studies on the subspecies of Brachymystax lenok tsinlingensis, China. J Lanzhou Univ 20:92-95 (in Chinese with English Abstract)

Wang K, Zhang S, Wang D, Xin M and others (2015) Development of 27 novel cross-species microsatellite markers for the endangered Hucho bleekeri using next-generation sequencing technology. Conserv Genet Resour 7: 263-267

Y Yu JN, Kwak M (2015) The complete mitochondrial genome of Brachymystax lenok tsinlingensis (Salmoninae, Salmonidae) and its intraspecific variation. Gene 573: 246-253

Yue PQ, Chen YY (1998) China red data book of endangered animals, Pisces. Science Press, Beijing (in Chinese)

* Zhang CL, Tong GX, Kuang YY, Zhang C, Yin JS (2010) Applicability of microsatellite DNA markers to the parental identification of Hucho taimen (Pallas). Zool Res 31:395-400 (in Chinese with English Abstract)

Zhao YH, Zhang CG (2009) Threatened fishes of the world: Brachymystax lenok tsinlingensis Li, 1966 (Salmonidae). Environ Biol Fishes 86:11-12

Submitted: February 1, 2016; Accepted: March 30, 2017

Proofs received from author(s): May 12, 2017 